\title{
A Method to Evaluate Human Spatial Coordination Interfaces for Computer-Assisted Surgery
}

\author{
M.A. Cardin, J.X. Wang, and D.B. Plewes \\ Department of Imaging Research, \\ Sunnybrook and Women's College Health Sciences Centre, \\ 2075 Bayview Avenue, Toronto, Ontario, Canada \\ \{ma.cardin, jxwang, don.plewes\}@swri.ca \\ http://www.swri.ca/groups/dbp
}

\begin{abstract}
Computer assistance for breast conserving surgery requires a guidance method to assist a surgeon in locating tumor margin accurately. A wide array of guidance methods can be considered ranging from various pictorial representations, symbolic graphical interfaces as well as those based on other sensory cues such as sound. In this study, we present an experimental framework for testing candidate guidance methods in isolation or in combination. A total of 22 guidance approaches, based on stereographic, non-stereographic, symbolic and auditory cues were tested in a simulation of breast conserving surgery. Observers were asked to circumscribe a virtual tumor with a magnetically tracked scalpel while measuring the spatial accuracy, time and the frequency with which the tumor margin was intersected. A total of 110 studies were performed with 5 volunteers. Based on these findings, we demonstrated that a single view of the tumor with a stereo presentation in conjunction with an auditory guidance cue provided the best balance of accuracy, speed and surgical integrity. This study demonstrates a practical and helpful framework for testing guidance methods in a context dependent manner.
\end{abstract}

\section{Introduction}

Increasing interest in alternatives to conventional surgery for cancer applications has prompted a wide array of potential solutions, ranging from remote surgery under image driven, laparoscopic or robotic manipulation [1, direct real-time image guidance integrated into a more traditional surgical setting and the use of pre-operative imagery to track surgical maneuvers based on some model of tumor geometry 2] 3]. Regardless of the approach, the need to integrate spatial information into a surgical decision making framework poses significant challenges to the engineer and the surgeon alike. This is particularly challenging when the surgeon attempts to integrate virtual data seamlessly into their appreciation of the surgical field. Ideally, one hopes to deliver the maximum amount of spatial information to the surgeon without inducing fatigue or being overwhelmed by 
excessive data. As each surgical task poses differing challenges, this balance will depend on the surgical context.

We have been exploring one such task which aims to integrate MRI information for the purpose of breast conserving surgery (BCS). Our approach is based on a segmented model of a breast tumor for resection which is coordinated to the patient's actual tumor location and geometry during surgery. The surgical task of BCS is to ensure complete removal of all cancerous tissue while sparing normal tissue. In this paper, we consider the challenge of creating a surgeon-computer interface that achieves the desired goal of guiding the surgical maneuvers while not burdening the surgeon with excessive and potentially distracting information.

Choices for surgical interfaces range from purely visual to those which attempt to integrate other perceptual cues. In addition to the inclusion of stereo presentation of visual data, other synthetic options include the use of tactile and auditory cues along with symbolic directives which aim to lead the surgeon in their task. These have the advantage of being used in isolation or in hybrid combination with virtual imagery with the hope of improving information transfer. However, it is often unclear which interface method or combination thereof is most effective. In this work, we attempt to address this question and demonstrate a surgical interface simulator which measures the efficacy of differing interfaces in isolation or in hybrid combinations.

\section{Method}

A surgical platform was constructed which allowed reproducible positioning of a tracking system (Figure 1(a) in which a magnetic system was used to dynam-

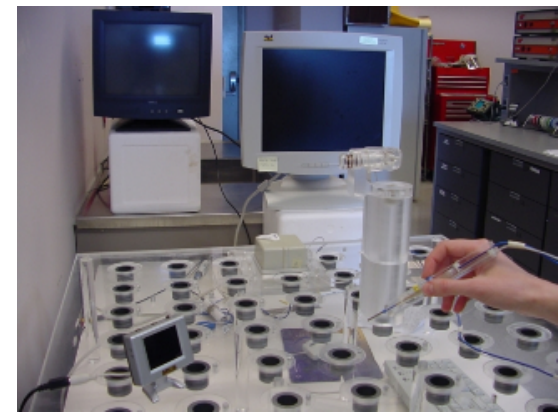

(a)

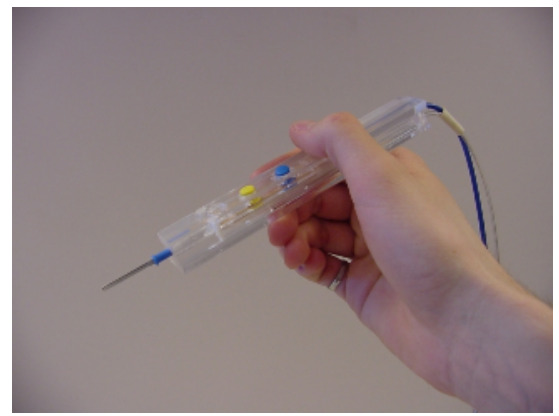

(b)

Fig. 1. a) Experimental setup showing the positioning table offering precise 6 DOF positioning and orientation of the magnetic sensor. The mini-viewer seen on the left is used in some of the various interface combinations, together with the LCD and CRT monitors at the back. The modified scalpel containing another magnetic sensor is shown on the bottom-right. b) Modified radio frequency scalpel that contains the magnetic sensor. 
ically locate a scalpel with respect to the boundaries of a virtual "tumor". The object of the experiment was to move the tip of the scalpel in a well-defined trajectory to simulate tissue cutting while recording the scalpel position. To achieve this, a $2 \mathrm{~mm}$ catheter-based, 6 DOF magnetic tracking probe (Ascension Technology) was embedded in a modified scalpel (Figure 1(b)). The desired trajectory was defined and fixed in space with another channel of the magnetic tracking system. The specific task was to trace out a circular path of radius of $200 \mathrm{~mm}$ while measuring the time taken to perform the task as well as the differences in the actual scalpel path relative to the desired path (i.e. the virtual tumor boundary). The operator was shown the "tumor" shape in various orientations as defined by the surgical platform.

In this experiment, we studied a number of visual and synthetic interface approaches. In the next, section we describe each of these approaches and the rationale behind their choice.

\section{$2.1 \quad$ Interface Options}

There were a total of 6 interface options we considered for this study. While many are possible, we chose these on the basis of our own experience and evidence from previous literature.

Interface 1 - Visual model of the scalpel and the desired task are viewed from a single viewport on a LCD monitor. This is shown in Figure 2(a) and represents the simplest visual presentation of the combined tracking task and the scalpel together. Motion of the scalpel is reflected by motion of the virtual scalpel in this presentation.

Interface 2 - Visual model of the scalpel and the desired task are shown from three orthogonal viewports together with a perspective rendering on the LCD monitor. This is shown in Figure 2(b) and provides complete threedimensional presentation of the tracking task and the scalpel motions from three orthogonal points of view and a single perspective viewpoint. This was the most complex visual model we tested.

Interface 3 - A stereo presentation of the task on a CRT monitor with the observer wearing optical shutter glasses (Stereographics) as shown in Figure 2(c), By this means, the observer perceived a clear three dimensional perception of the tracking task and the scalpel.

Interface 4 - In this case, we provided the observer with an auditory cue in which the amplitude of a tone with constant frequency increased as the probe approaches the tumor boundary. Another element of this sound guidance interface is noted by a change in frequency when the scalpel was moved to a position inside the tumor, indicating an error in resection. This interface is shown in Figure 2(d).

Interface 5 - In this case, we generated a small, "navigation compass" viewport which was presented on the LCD monitor. The compass operates to dynamically point in a direction that the scalpel should move to approach the desired position 


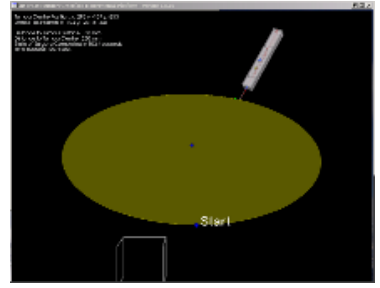

(a)

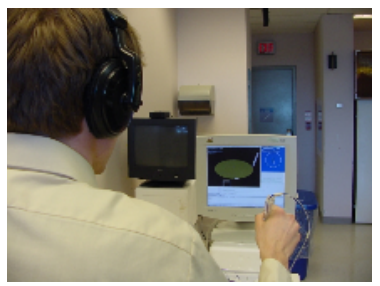

(d)

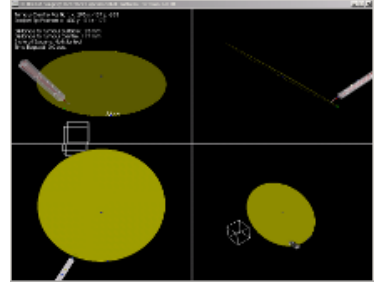

(b)

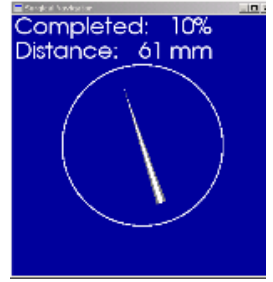

(e)

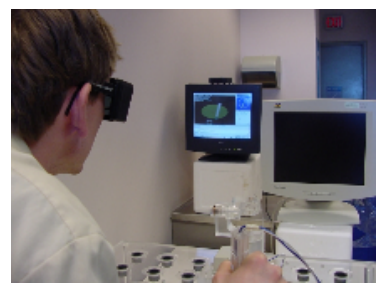

(c)

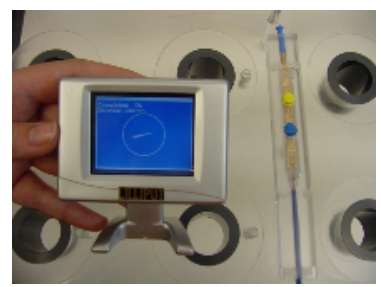

(f)

Fig. 2. a) Interface 1: one viewport with axial view and virtual scalpel. b) Interface 2: four viewports with axial, sagittal, coronal and a perspective view. c) Interface 3: stereo visualization on CRT monitor. d) Interface 4: Sound guidance and other visualization combination. e) Interface 5: Navigation compass. f) Interface 6: Navigation compass on mini-viewer and modified scalpel.

in a plane corresponding to the plane of trajectory. If the tip of the scalpel was found to be "above" the desired plane, the needle would increase in length. Likewise if it was below the desired level, the needle would shrink. The interface is shown in Figure 2(e). This was an attempt at a symbolic interface that was not pictorial but still provided all directional information for surgical guidance.

Interface $\boldsymbol{6}$ - In this interface, we explored the effect of "perceptual discontinuity" where the observer must constantly redirect their attention toward a monitor to gather perceptual information and away from the surgical area. To overcome this effect, we used a "mini-viewer" which shows the compass of interface 5, but placed immediately beside the surgical field so that the user does not need to look away from the surgical field when using it. This is shown in Figure 2(f)

\subsection{Volunteer Studies}

These six interfaces were studied in isolation and in combination. Table 1 shows the combinations of interfaces applied in this study. A total of 22 experiments were performed with varying combinations thought to provide interesting combinations of guidance methods. Each of the 22 experiments was performed with differing orientations of the virtual tumor, thus providing a diverse set of surgical trajectories. The volunteers were asked to move the scalpel around the virtual tumor boundary as accurately as possible. They were also informed not to move 
the scalpel to a point "inside" the tumor, as this would reflect a surgical error corresponding to degrading the tumor margin for histology. They were also told to complete the task as quickly as possible and that they were being timed. The balance between speed and accuracy was left to the volunteer. This experiment was repeated for five volunteers who were asked to complete all the experiments in a single sitting. The order of experiments was determined randomly and a total of 110 experiments were completed.

Each volunteer was asked to move the scalpel around the desired path assisted by the interface combination for that experiment. The positional error, the total time to encompass the path and the frequency with which the scalpel was found to "cut into" the tumor were all measured.

\subsection{Measures of Surgical Efficiency}

For each experiment, the distance between the surgical path and the actual tumor boundary was measured at all times with the magnetic trackers. The positional error was evaluated as the closest distance between the tip of the virtual scalpel and the virtual tumor boundary. From this data a root-meansquare (RMS) distance error was determined. The time taken to complete the task was also measured by providing a target on the virtual tumor boundary to keep track of the state of progression of the virtual surgery. This target point would advance on the circular path each time the closest point to the scalpel tip would cross the previously determined target point, thus simulating a virtual "cutting" of the tumor. The task was completed when the target point had swept over all point locations on the circular path. We also recorded the fraction of distance measurements that were positioned inside the virtual tumor. This error was recorded whenever a part of the virtual scalpel was touching the virtual tumor from the inside. It was recorded as a negative distance measurement to the scalpel tip, as seen on Figures $3(\mathrm{a})$ and $3(\mathrm{~b})$.

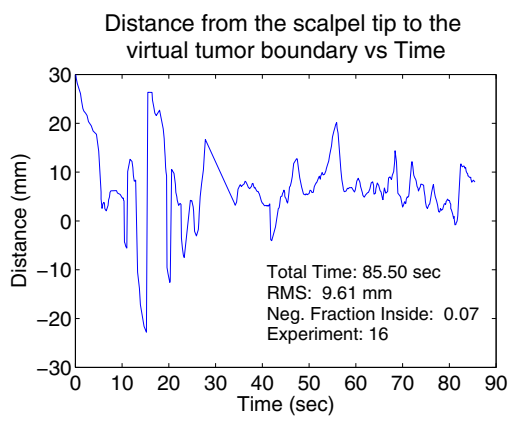

(a)

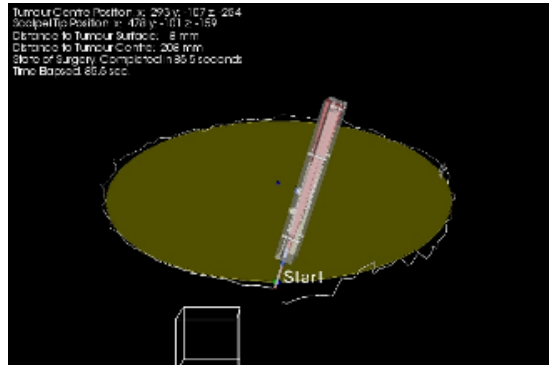

(b)

Fig. 3. a) Typical positional error as a function of time for completion of experiment 16. The total time in seconds to complete the surgical task, RMS distance and fraction of distance measurements taken inside the tumor are also shown. b) Surgical path traced out by one volunteer in experiment 16 . 


\section{Results}

For all experiments described in Table 1, the average RMS distance was calculated across all 5 volunteers for each experiment. In addition, the average time to completion and the average percentage of distance measurements recorded inside the tumor were also considered (Figure 44). We can see from these data considerable variation in these parameters across each experiment. In general, we see a slight advantage for experiment number 16 in which the mean value is

Table 1. The combination of interface studies in the virtual surgical task

\begin{tabular}{|c|c|c|c|c|c|c|c|c|c|c|c|c|c|c|c|c|c|}
\hline Method of Guidance & \multicolumn{17}{|c|}{ Experiment Number } \\
\hline & & $\overline{234}$ & 56 & 789 & 10 & 11 & $\overline{12}$ & $\overline{13}$ & $\overline{14}$ & 15 & 16 & & 18 & & 20 & & $\overline{22}$ \\
\hline Single Viewport on LCD & $\mathrm{x}$ & $\mathrm{x}$ & $\mathrm{x}$ & $\mathrm{x} x$ & & $\mathrm{x}$ & & $\mathrm{x}$ & $\mathrm{x}$ & $\mathrm{x}$ & $\mathrm{x}$ & $\mathrm{x}$ & $\mathrm{x}$ & & & & \\
\hline Four Viewports on LCD & & $\mathrm{x} \quad \mathrm{x}$ & $x$ & $x$ & $\mathrm{x}$ & & $\mathrm{x}$ & & & & & & & & & & \\
\hline Stereo on LCD & & & & & & & & $\mathrm{x}$ & $\mathrm{x}$ & $\mathrm{x}$ & $\mathrm{x}$ & $\mathrm{x}$ & $\mathrm{x}$ & & & & \\
\hline Sound Guidance & & & & $\mathrm{x} \times \mathrm{x}$ & $\mathrm{x}$ & $\mathrm{x}$ & $\mathrm{x}$ & & & & $\mathrm{x}$ & $\mathrm{x}$ & $\mathrm{x}$ & & & $\mathrm{x}$ & $\mathrm{x}$ \\
\hline Compass on LCD & & $\mathrm{xx}$ & & $\mathrm{x}$ & $\mathrm{x}$ & & & & $\mathrm{x}$ & & & $\mathrm{x}$ & & & $\mathrm{x}$ & & $\mathrm{x}$ \\
\hline Compass on "Mini-viewer" & & & $\mathrm{x} x$ & & & $\mathrm{x}$ & $\mathrm{x}$ & & & $\mathrm{x}$ & & & $\mathrm{x}$ & $\mathrm{x}$ & & $\mathrm{x}$ & \\
\hline
\end{tabular}

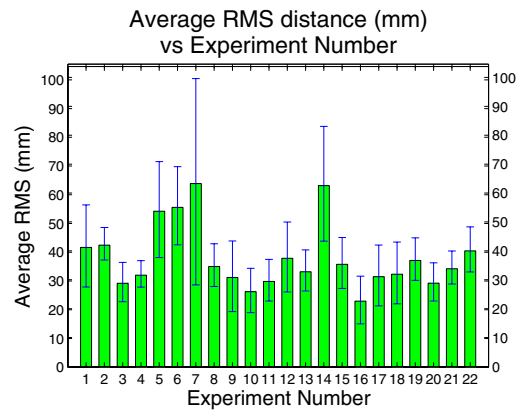

(a)

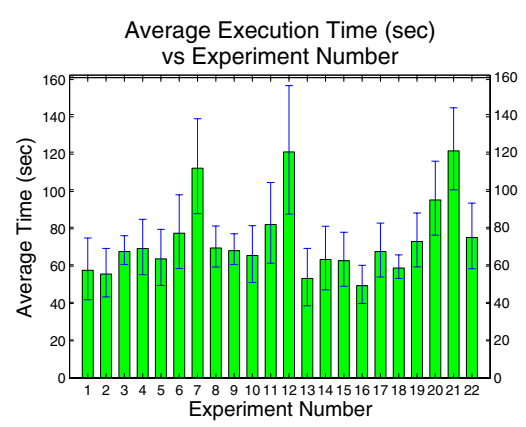

(b)

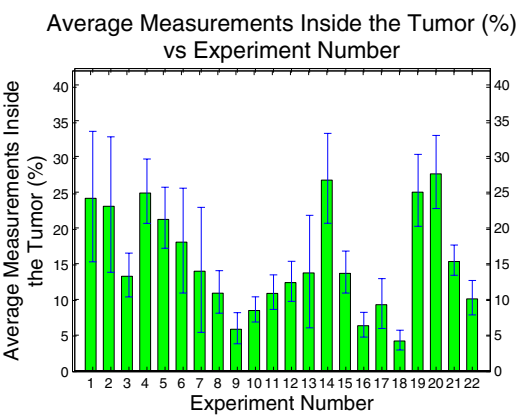

(c)

Fig. 4. The RMS error (a), the time to complete the task (b) and the frequency with which the scalpel cut into the tumor (c) versus the guidance method (see Table 10). The variation is the standard error of each parameter for the five observers. 
minimized for RMS error and time. In addition, this same method also shows a low value for the frequency with which the scalpel ventured inside the tumor. However, in this latter case, experiments 9 and 18 also performed well. However, methods 9 and 18 did not perform as well with regard to spatial error and time.

On the basis of these pilot data, we determined that the guidance method tested in experiment 16 was the most successful approach. This guidance method involved the combination of a one viewport with the additional support of $3 \mathrm{D}$ stereography and auditory guidance. It is noteworthy that when the compass was provided in the mini-viewer, there appeared to be no significant advantage with regard to error and time but showed a slight benefit in terms of cutting into the tumor.

\section{Discussion}

These studies demonstrate that there is an optimum combination of guidance approaches for the guidance tasks presented in this paper. We note that providing as much imaging information does not necessarily increase the ability of the user to use this information. Furthermore, the use of stereo visualization was not always seen to be helpful. Rather, we found that the use of stereo data needed to be done in conjunction with other approaches to have a significant effect on its overall utility. Another interesting finding is that the use of the navigation compass did not contribute efficiently in improving the results. Furthermore, the degradation from "perceptual discontinuity" was not evident in these data as indicated by the use of the mini-viewer for the compass guidance as compared to the compass presented on the monitor. This does not mean that the notion of perceptual discontinuity was not operational in this study, but may reflect that use of a compass guidance approach did not appear to offer much advantage. In contrast, the use of an auditory cue was found to be of value when offered in conjunction with visual cues.

These general observations are corroborated by the comments received from volunteers while performing the experiments. They outlined that four viewports generally added on the complexity of the task while not providing additional support to improve performance. Volunteers would still resort to one or two viewports to complete the task. In addition, the results reflect a general appreciation of the sound guidance system, mainly for the evaluation of depth in performing the task. Volunteers also mentioned the difficulty in using the navigation compass, which is also reflected by it not having a large impact on the accuracy data.

\section{Conclusion}

This experimental method has been developed to test the efficacy of varying guidance methods for computer-assisted surgery. We tested a range of guidance methods which have been chosen to represent appropriate and potentially useful candidates for virtual surgical guidance. Our goal was to study the effect 
of combining guidance cues in a quantitative and controlled setting with the objective of seeking approaches which maximized the accuracy and speed of an intervention without burdening the user. We found, considerable variation in the utility of these approaches and that stereo and auditory guidance appeared to be a fruitful option. Clearly, other methods could have been chosen and this same experimental platform can be used to evaluate these. In the future, we will be using this approach to test other guidance methods with increased degree of complexity as we move toward developing a computer assisted surgical methods for breast conserving surgery.

\section{References}

1. Riva, G.: Applications of virtual environments in medicine. Methods of Information in Medicine 42(5) (2003) 524-534

2. Shuhaiber, J.: Augmented reality in surgery. Archives of Surgery 139 (2004) 170 174

3. Tamaki, Y., et al.: Intraoperative navigation for breast cancer surgery using 3d ultrasound images. Computer Aided Surgery 4 (1999) 37-44 